\title{
Comparison of SOI Power Device Structures in Power Converters for High-Voltage, Low-Charge Electrostatic Microgenerators
}

\author{
Bernard H. Stark and T. C. Green
}

\begin{abstract}
An inertial generator based on a moving-plate capacitor can provide energy for medical sensors from low-frequency (human body) motion. The energy exists as a very small charge $(4 \mathrm{nC})$ at high voltage $(300 \mathrm{~V})$. An initial proposal for power processing used a carefully scaled lateral power MOSFET-diode pair, which results in a low, but sufficient, energy yield. It was found that increasing the area of the MOSFET to reduce conduction loss is highly detrimental to the energy yield because of capacitive loading of the generator. This paper examines alternative device topologies which may greatly increase the energy yield for a given system size by increasing both the generation efficiency and the conversion efficiency. An insulated gate bipolar transistors (IGBT) and a MOS-triggered thyristor, both based on previous silicon-on-insulator MOSFET designs, are examined for their switching speed and losses using physics-based finite-element simulation. The scaling criteria to achieve optimum system effectiveness are discussed. The small charge available from the generator results in a brief conduction period which does not allow the devices to reach their steady-state carrier distributions. Nevertheless the IGBT, and especially the MOS-triggered thyristor, switch on faster than the MOSFET, run at higher current densities, and provide improved efficiency. This allows the devices to be reduced in area leading to less capacitive loading on the generator. It also allows a reduction in the value and volume of the circuit inductor without a onduction loss penalty. We describe the device behavior in detail for the various phases of the conversion cycle and illustrate device/circuit tradeoffs graphically. Requirements are outlined for the development of power devices for microgenerators in implanted medical sensors.
\end{abstract}

Index Terms-Energy scavenging, insulated gate bipolar transistors (IGBTs), microelectromechanical systems (MEMS), microgenerators, MOS controlled thyristor, MOSFETs, pulse power switches, silicon-on-insulator (SOI).

\section{INTRODUCTION}

M ICROMACHINED power generators may one day enable the realization of self-powered microsensor-transmitter systems. Such systems would contain a power source, power conditioning, sensors, signal processing and data transmission, all integrated onto a single silicon chip. One important application is the field of implantable biosensors where the aim is to transmit chemical concentrations or pressures in the blood stream at a low data rate to a handheld monitoring device. This

Manuscript received October 12, 2004; revised March 15, 2005. This work was supported in part by the EPSRC and in part by the DC initiative (EU Framework V) under the ORESTEIA Project. The review of this paper was arranged by Editor M. A. Shibib.

The authors are with the Department of Electrical and Electronic Engineering, Imperial College London, London SW7 2AZ, U.K. (e-mail: b.stark@ imperial.ac.uk).

Digital Object Identifier 10.1109/TED.2005.850940 would, for example, enable tighter glucose control which would reduce the suffering caused by diabetes. Implantable biosensors have also been suggested for continuous monitoring of blood pressure in patients with hypertension, as this can significantly increase medication compliance. Real-time processing of electrocardiograph traces can be very effective at revealing the early stages of heart disease [1], [2].

Implanted sensors may require intrusive surgery and would therefore need to remain in place for many years. A probable way of powering these devices is through scavenging energy from the environment, particularly from the motion of the devices themselves. An extensive review of suitable micromachined microelectromechanical systems (MEMS) microgeneration methods is given in [3].

Early work in energy scavenging has focussed on electromechanical generation. Initial studies have shown [4] that the power processing circuit has some difficult constraints that require significant innovation to reach a usable solution. An important area for innovation is tailoring the power semiconductor devices for the unusual voltage, current, charge, and time conditions that exist.

\section{SYSTEM DESCRIPTION}

The microgenerator system discussed uses the relative motion between a proof mass and a frame to do work against an electrostatic field. It uses the acceleration available from body motion [5] which is irregular, and therefore the generator considered here is of a nonresonant electrostatic type [6]. A prototype generator [7] of the type investigated is shown in Fig. 1.

The moving mass occupies most of the generator area and it is loosely sprung, so that it can be stimulated from low-frequency, irregular movement. The exploded view in Fig. 2 shows the generators three micromachined layers; the center frame that suspends the moving plate which is free to move vertically, the base holding a stationary capacitive plate and charging electrodes, and a lid containing discharge contacts. This structure has been chosen due to its compatibility with a CMOS process.

Instead of using discharge contacts, it is important to implement the energy extraction via a converter, as this allows the use of control methods [6] optimized for extracting maximum energy from slow variable human body motion as opposed to low-amplitude high-frequency machine vibration.

The electrostatic generation method [8] requires the plates to be precharged, which creates an attracting electrostatic force holding the plates at their closest. The charging circuit is then detached from the moving plate and the device is primed and 


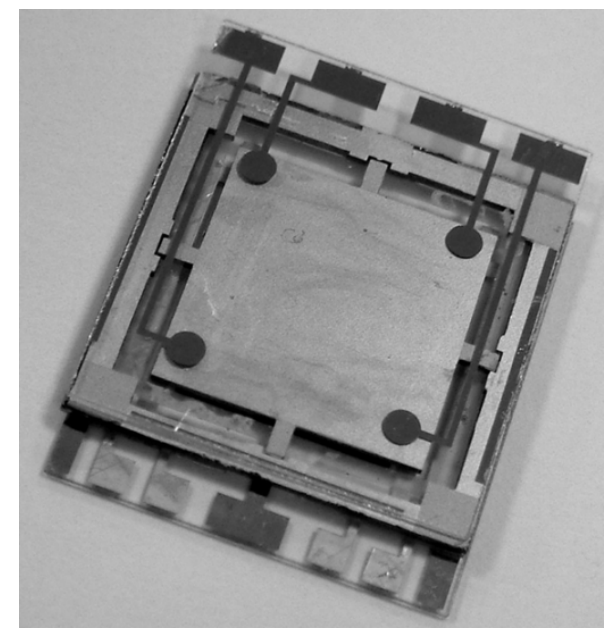

Fig. 1. Prototype generator $(20 \times 20 \times 1.5 \mathrm{~mm})$.

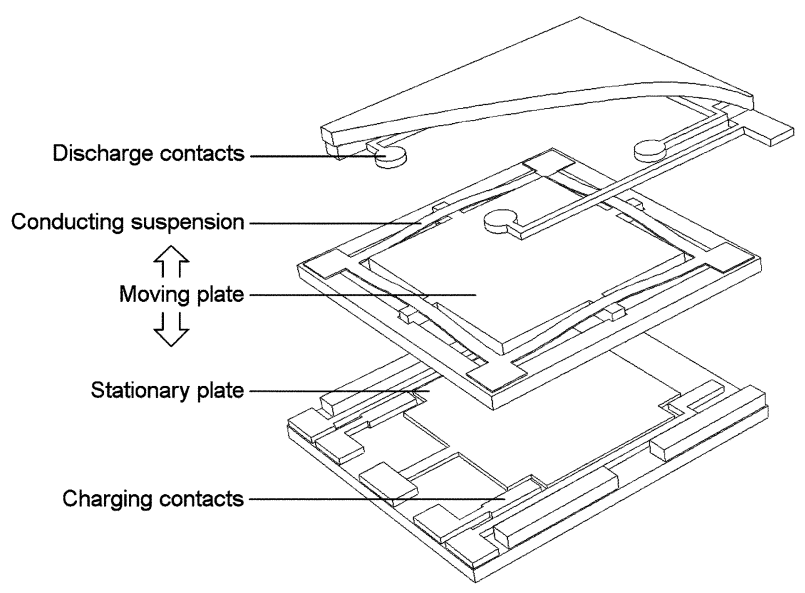

Fig. 2. Exploded view of the generator in Fig. 1. The charging contacts are slightly thicker than the stationary capacitor plate, and this height difference isolates the plates from each other at minimum separation.

ready to generate. When the movement of the device reaches the point where the acceleration $\times$ mass of the moving electrode exceeds the electrostatic force holding the plates together, the movable plate breaks away and moves against the attracting force between the plates, thereby increasing the potential energy in the system. If the charge $q$ is held constant, the capacitor voltage increases, and the increase in potential energy is given by

$$
E=\frac{1}{2} q\left(V_{2}-V_{1}\right) \quad \text { or } \quad E=\frac{1}{2} V_{2}^{2} \frac{C_{2}}{C_{1}}\left(C_{1}-C_{2}\right)
$$

where $V_{1}$ is the precharge voltage, $V_{2}$ is the finishing voltage, and $C_{1}$ and $C_{2}$ are the start (closed) and finishing (open) capacitances of the generator. It can be seen that, in order to obtain a good energy yield, it is beneficial to arrange for a high final voltage on the capacitor plates.

\section{Semiconductor Device ReQuirements}

\section{A. Required Devices}

At full plate separation the voltage of the prototype generator rises to around $V_{2}=300 \mathrm{~V}$ and the capacitance from which the energy is to be extracted is reduced to approximately $C_{2}=10 \mathrm{pF}$. Fig. 3 shows a half-bridge step-down (or buck-)

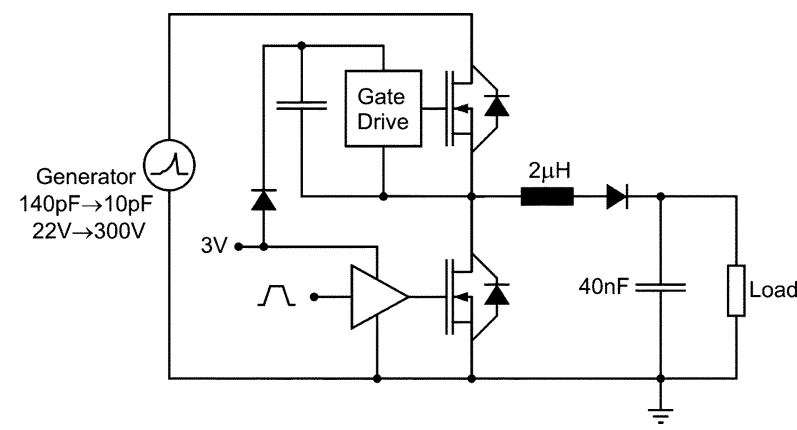

Fig. 3. Half-bridge step-down (or buck-) power converter with boot-strap high-side gate drive. The low-side MOSFET is only required for the boot-strap gate-drive.

converter circuit which can discharge the generator and convert the extracted energy to a useful output voltage of around $3 \mathrm{~V}$.

There are three high-voltage semiconductor devices required. The high-side branch requires a switch such as a MOSFET rated for the full generator voltage and which can carry the peak conversion current. The low-side requires a diode to conduct the peak conversion current and a parallel MOSFET which is only switched on to feed the boot-strap gate-drive while the generator voltage is zero. From here onwards we ignore this parallel MOSFET, because it can be scaled much smaller than the diode, and treat this circuit as having one high-side switch and one low-side diode.

\section{B. Generator Compatibility}

An important consideration is that the converter should not significantly leak current once a high voltage has been generated, or present a large parasitic capacitance that absorbs charge from the generator during the movement of the plate.

A high-side 330-V SOI MOSFET and a low-side 330-V SOI diode have been shown to have sufficiently low leakage and parasitic characteristics [4]; as long as the active areas of the two power semiconductor devices are $0.45 \mathrm{~mm}^{2}$ or below, then only $13 \%$ of the generator charge is lost to the parasitic capacitance of the semiconductors devices during the flight of the plate. For ease of comparison we equate this area to 30 unit "cells." Doubling the area of the devices increases the charge loss to $26 \%$ and the associated energy loss to $46 \%$. The requirement to limit the energy used in precharge means that this charge loss must be constrained and this unfortunately limits the MOSFET area to around 30 cells.

\section{Requirements Relating to Conversion}

Buck converters convert an amount of charge at a high voltage to a larger amount of charge at a low voltage. They typically operate in two phases: first the device is switched on and the current ramps up in the inductor (i.e., magnetization), and then the device switches off and the current free-wheels through the diode (demagnetising the inductor). During both phases the output capacitor is being charged.

In this application the operation is more complex. First, it is the devices' response in the first $10 \mathrm{~ns}$ after applying the switching signal which defines the circuit behavior. The circuit is operating at one $60 \mathrm{~ns}$ shot per second which is a much shorter duty-cycle than is usual in power circuits. Second, the switch 


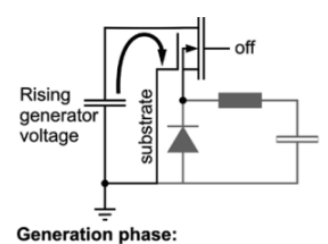

Generation phase:

Switch: Charg: -
Diode:

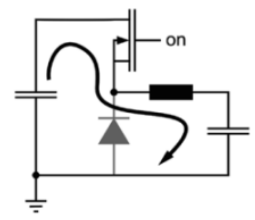

Magnetisation phase:

Switch: Turn-on still in progress Diode: Discharges through inductor

Fig. 4. Four phases of operation and their distinct current patterns: A generation phase and three phases of conversion.

requires no turn-off capabilities because the charge on the generator is completely extracted in under $10 \mathrm{~ns}$, causing the switch current naturally to drop to zero. Third, the devices are run at a very high current density and high forward voltages in order to keep the device area low to reduce the charge loss and associated generator inefficiency.

Operation using very short pulses, in which the devices may not reach their steady-state on-state condition and where high current densities occur, was found to be most efficient form of operation. As a consequence, standard MOSFETs are not appropriate and we need to tailor the devices to this application.

It is convenient to split the operation of the circuit into four phases as shown in Fig. 4. In the first phase, the switch is off and the generator voltage is rising. It is important that the switch has a small capacitance, or else the generator will lose its charge to the switch and not generate sufficient energy. In the second phase, during turn-on of the switch, current flows into the diode to establish a reverse bias and to allow the voltage over the switch to collapse. This current is supplied by the generator and is therefore an unwanted loss of charge. A low switch on-state voltage is required, to minimize conduction losses, and a low diode capacitance reduces the amount of charge lost. During the third phase, the inductor current increases and the generator voltage falls until the generator is completely discharged. At this point, the inductor current is at its maximum. Again, a low on-state voltage is required for the switch. Then, the longest phase begins, in which the current free-wheels through the diode. Here the diode on-state is important. It is clear that there are opposing requirements, and therefore an optimum tradeoff needs to be sought.

\section{Requirements Relating to System Integration}

The buck converter using 30-cell devices has been shown to have a conversion efficiency of around 40\% [4], however this is only obtained if the current pulses are kept relatively long and of low amplitude using a $10 \mu \mathrm{H}$ inductor. Larger devices lower the efficiency due to increased charge sharing during turn-on, and smaller devices lower the efficiency through higher conduction losses. In addition the diode area needs to be optimized.

Unfortunately a $10 \mu \mathrm{H}$ inductor is difficult to integrate due to its physical size and due to constraints that must be placed on its

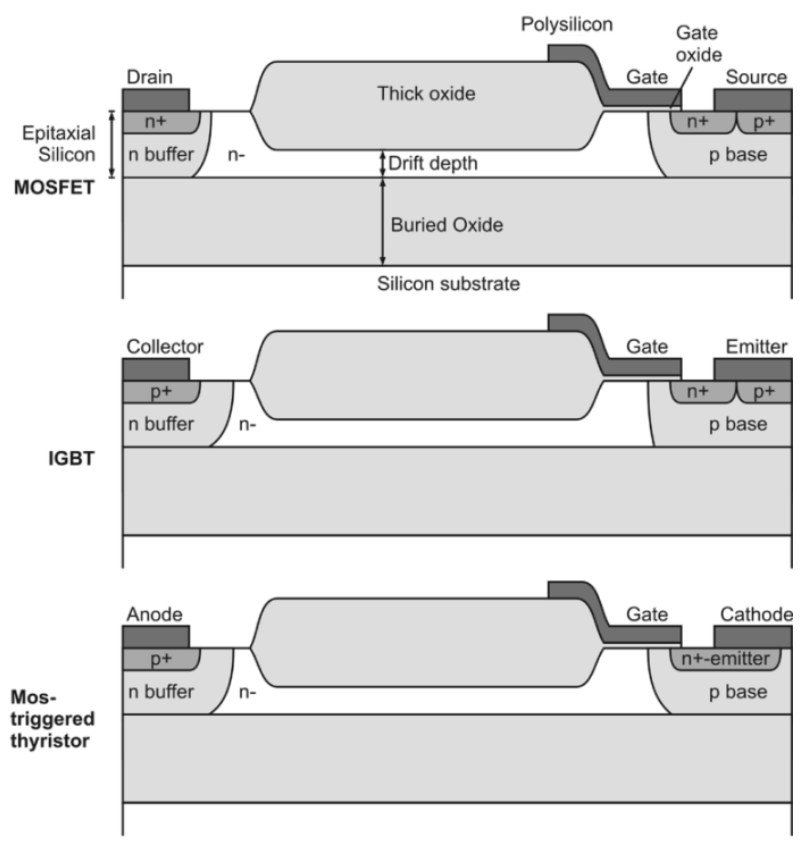

Fig. 5. Progression from a MOSFET to a MOS-triggered thyristor, via an IGBT. To obtain the device structure the cross sections are mirrored at the drain and extruded into third dimension. Section width: $70 \mu \mathrm{m}$, epitaxial silicon thickness: $1.4 \mu \mathrm{m}$, buried oxide thickness: $4 \mu \mathrm{m}$. All three devices have graded n-dopings.

parasitic capacitance [9]. It is preferable to use a lower inductor value. An inductor of $2 \mu \mathrm{H}$, however, shortens the conversion cycle and increases the current amplitude. As a consequence, the efficiency of a MOSFET power conversion circuit would drop to around $13 \%$. We therefore investigate other device topologies which are known to achieve higher current densities.

\section{DeVICE DESIGN}

\section{A. Initial Device Concepts}

To achieve CMOS compatibility and the very high insulation levels required, a thin-layer silicon-on-insulator (SOI) process [10] was chosen for the switching device and diode. Initially, a MOSFET was considered to be adequate and a trial design was conducted [4]. It was not obvious that a high-level injection device implemented in thin SOI would show much improvement over a MOSFET, which shows no conductivity modulation delay, because of the very short conversion cycle (max $\cdot 60$ ns). In addition, the benefits of high-level injection may be impeded by the very thin drift region and high drift resistance of an SOI design. However, due to the strict limitation on the device area, it is not possible to operate the MOSFET in its efficient linear region and it operates partially in saturation. This motivated the design of an IGBT and a thyristor (in the same process) to investigate the usefulness of conductivity modulation in this application.

Fig. 5 depicts cross sections of the three devices used in this paper. The MOSFET was designed according to process specifications in the literature [10] to block $330 \mathrm{~V}$ and carry a current of $1 \mathrm{~mA}$. The MOSFET was then modified to create an IGBT, and further changes to the masking result in thyristor structures.

The IGBT and thyristor both have $\mathrm{p}^{+}$-emitters at the anode which inject holes into the $\mathrm{n}^{-}$-region. $\mathrm{N}$-buffers are required to 
shape the electric field to avoid premature breakdown, although these should be as weak as possible to maximize the injection efficiency of the anode.

In IGBTs, the hole current flowing around the emitter $\mathrm{n}^{+}$-well causes a lateral voltage drop along this well which can, beyond the latch-up current limit, forward bias this $n^{+}$-p junction. At this instant electrons are injected from the $\mathrm{n}^{+}$-region directly into the device, bypassing the gates $\mathrm{n}$-channel. This increased "base current" into the $\mathrm{p}^{+} \mathrm{n}^{-} p \mathrm{p}$-transistor causes more holes to flow, which, in turn, increases the electron injection from the $\mathrm{n}^{+}$-emitter. The device latches up and achieves a lower on-state voltage at the expense of loss of gate control. Therefore the IGBTs p-base requires sufficient depth and doping concentration in order to reduce the lateral resistance. The latching current level drops with rising device voltage, because the path for hole current through the p-base becomes restricted as the p-base is depleted. In other words, the IGBT has a high latching current to ensure safe turn-off. In this application, turn-off is not required and therefore the on-state conductivity can be improved at the expense of the turn-off capabilities. Latching can be allowed since natural commutation occurs in the circuit and the latching reduces the conduction power loss. The latching current can be lowered and we call the resulting structure a MOS-triggered thyristor. It is important to note that this is not a MOS-controlled thyristor (MCT) due to its limited turn-off ability, although this MOS-triggered thyristor may be seen as an MCT stripped of the components which would only be required for safe turn-off. In practice, this means that the thyristor has a reduced p-base doping and reduced shorting. This is effected in part by increasing the width of the $\mathrm{n}^{+}$-well at the cathode (emitter) and by only shorting the p-base in islands, as opposed to having one continuous short aligned with the third dimension.

\section{B. System Simulation}

The devices are modeled using a physics based finite-element simulator (Silvaco). The converter circuit was simulated using one of the three different high-side devices and a single low-side diode, with different scaling factors. Accurate system simulation required the incorporation of detailed finite-element models of the semiconductors which account for the charge flows in the devices and in the substrate wafer, mainly because of the large influence that parasitic currents have on a system such as this which contains very little charge. Simulations were performed using Silvaco software in mixed-mode which combined finite-element models of the semiconductor devices and lumped models for the remainder of the circuit. The finiteelement models account for physical effects such as electronhole-pair generation and impact ionization (leakage currents), substrate currents (important for the high-side devices integrated onto grounded substrates) and charge storage. Carrier lifetimes of $1 \mu$ s were specified corresponding to nonirradiated epitaxial silicon.

It is important to simulate the two devices with the buried oxide and conducting substrate included because the capacitive currents through the high-side switch and substrate during turnon are significant compared to the amount of charge available on the generator. Combining finite-element level simulation of the semiconductors with the mechanical simulation was not judged necessary at this stage.

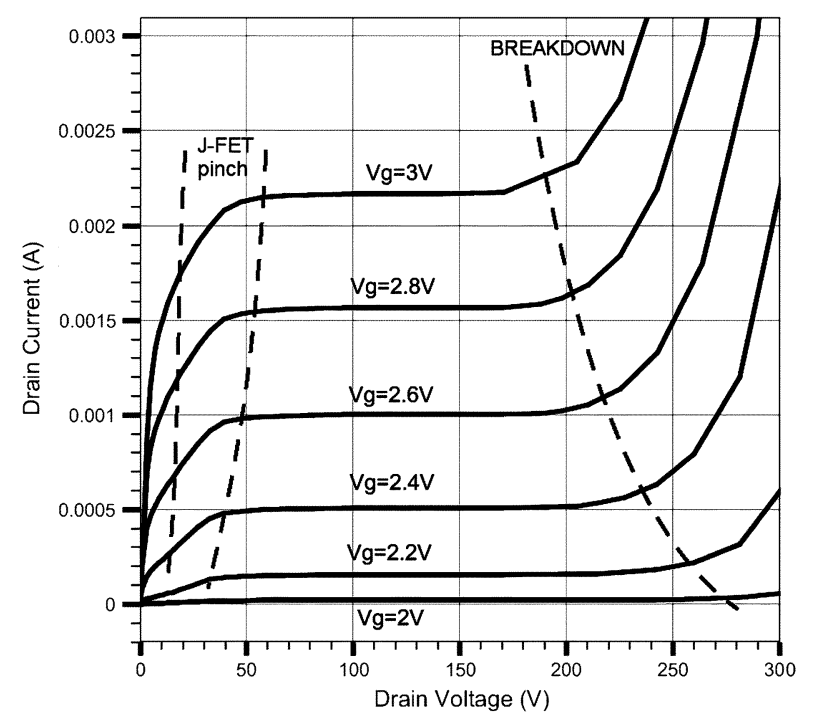

Fig. 6. Output characteristics obtained from the finite-element model of the MOSFET for one cell $\left(0.015 \mathrm{~mm}^{2}\right)$.

\section{MOSFET: Simulated Steady-State Output Characteristics}

The simulated output characteristics for one $0.015 \mathrm{~mm}^{2}$ MOSFET cell (Fig. 6) show the typical linear region (up to approximately $50 \mathrm{~V}$, the saturated region, and the region where the device begins to avalanche at high voltages. With the gate shorted to source the device blocks $330 \mathrm{~V}$.

The device was designed for a gate threshold voltage of $2.0 \mathrm{~V}$ such that it can be driven with $V_{\mathrm{GS}}=3.0 \mathrm{~V}$ from a gate-drive supplied by the output voltage of the circuit. This application requires little safety margin as there is not enough energy in the generator to cause avalanche damage if the device were to turn on prematurely. At $V_{\mathrm{GS}}=3.0 \mathrm{~V}$ the device saturates at just below $2 \mathrm{~mA}$.

In the off-state (for gate voltages below $2 \mathrm{~V}$ ) the high voltage on the MOSFET causes carrier-pair generation and a leakage current that is dependent on the voltage and the active area. At room temperature and $300 \mathrm{~V}$, a single cell conducts a leakage current of $98 \mathrm{pA}$.

\section{IGBTs Output Characteristics}

The IGBTs blocking characteristics and leakage current are very similar to that of the MOSFET because it uses an identical blocking junction. However, the cell has a much higher current carrying capacity. For a gate voltage of $3 \mathrm{~V}$ and a forward current of $2 \mathrm{~mA}$, the voltage drop is $2.4 \mathrm{~V}$. The MOSFET was beginning to saturate under the same conditions and had a voltage drop of $35 \mathrm{~V}$.

The output characteristics in Fig. 7 (to a different scale to Fig. 6) show much higher currents are achieved for a given voltage. Beyond a certain current (dependent on the gate voltage) the device saturates and then begins to avalanche. As the current increases further the device latches up.

Increasing the device voltage beyond $20 \mathrm{~V}$ causes advancing depletion regions to restrict the current flow (J-FET pinch) thereby reducing the gradient in the linear region and causing a negative gradient as the device begins to saturate. 


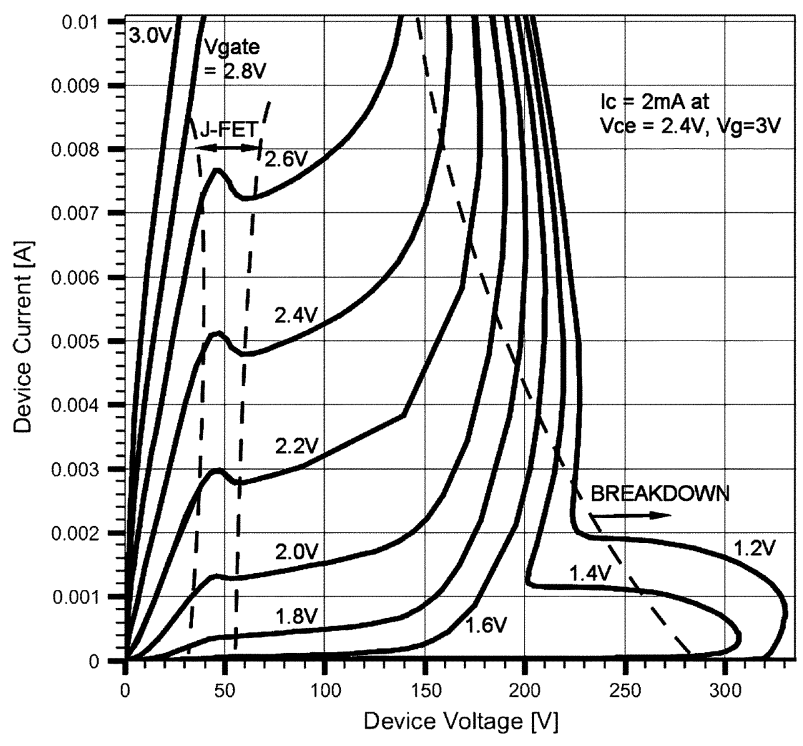

Fig. 7. Output characteristics obtained from the IGBTs finite-element model for one cell $\left(0.015 \mathrm{~mm}^{2}\right)$, and no self-heating.

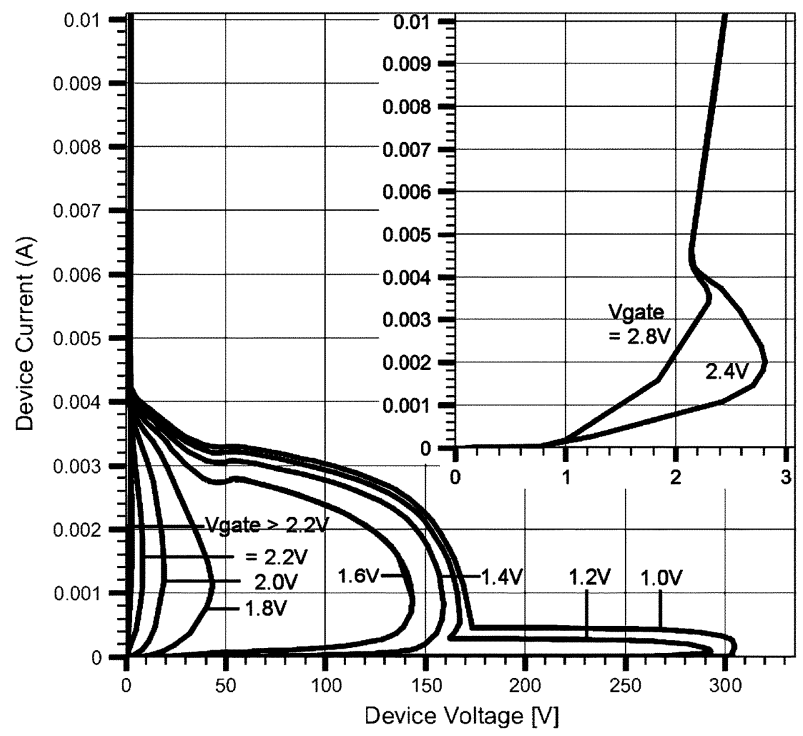

Fig. 8. Output characteristics obtained from the Thyristors finite-element model for one cell $\left(0.015 \mathrm{~mm}^{2}\right)$, and no self-heating.

\section{E. MOS-Triggered Thyristors Output Characteristics}

Simulated output characteristics of the MOS-triggered thyristor are shown in Fig. 8. They are similar to those of the IGBT up to the (voltage-dependent) current level at which the device latches up. At $300 \mathrm{~V}$, latch up occurs for less than 1 $\mathrm{mA}$, and at low voltage it occurs at around $2-3 \mathrm{~mA}$. With a gate voltage of $3 \mathrm{~V}$, the cell carries $9 \mathrm{~mA}$ for a voltage drop of $2.4 \mathrm{~V}$ compared to $2 \mathrm{~mA}$ for the IGBT. This can be seen in the expanded characteristics shown in the top-right in Fig. 8.

The reduction of on-state voltage drop achieved by moving from MOSFET to IGBT to MTT could have been achieved instead by increasing the area of the MOSFET. The advantage of using high level injection in a device of the same area was that the device capacitance in off-state was kept below the maximum value needed for effective generator operation.

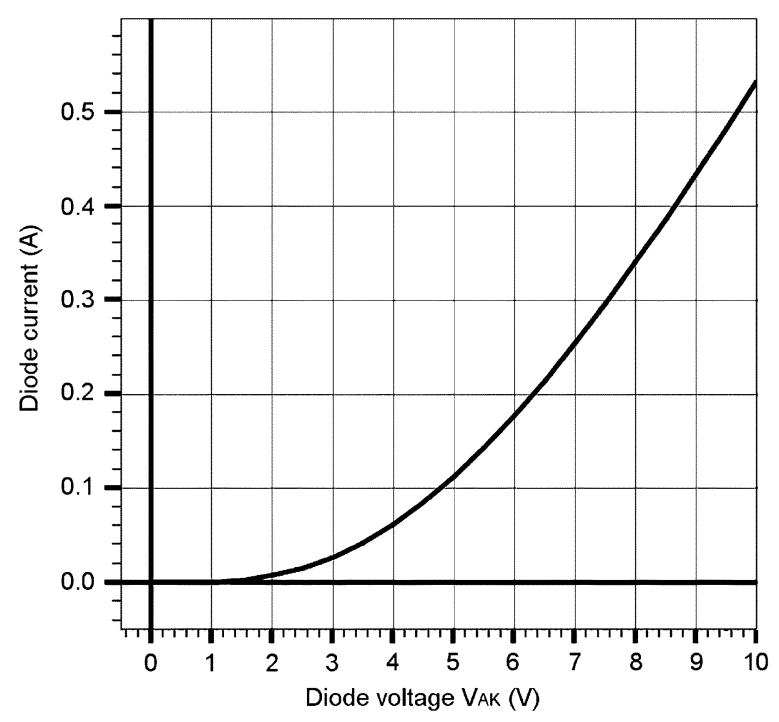

Fig. 9. $I-V$ characteristics obtained from the diodes finite-element model for one cell $\left(0.015 \mathrm{~mm}^{2}\right)$, and no self-heating.

A key design parameterof the MTT is the latching current level and it can be influenced by altering the shape and doping levels of the p-base and of the wells delimiting it. Care has to be taken that the gate threshold voltage is maintained.

\section{F. Diode Characteristics}

The finite-element diode model uses the same SOI process used for the three devices. The diode is a double-sided injection device similar to the thyristor, and therefore the steady-state current-voltage characteristics shown in Fig. 9 are almost identical to the output characteristics of the latched-up thyristor.

\section{Device Transient Performance}

It is important to note that, although curve traces provide some useful indication of the behavior of the devices, they are based on steady-state results that assume that the device has been maintained at each point for long enough (at least $1 \mathrm{~ms}$ ) for the carrier concentrations to stabilize. In this application, there is very little charge available in the generator and the entire conversion cycle takes around $60 \mathrm{~ns}$. The devices will not reach their steady-state carrier distributions and their behavior varies significantly from that indicated by their output characteristics. An assessment of the relative merits of various device designs must be based on a detailed finite-element model incorporated into a circuit simulation.

\section{A. Circuit Behavior Using the MOS-Triggered Thyristor}

The generator was modeled as a $10 \mathrm{pF}$ capacitor charged to $300 \mathrm{~V}$. The MOS-triggered thyristor is turned on $10 \mathrm{~ns}$ into simulation with a $3-\mathrm{V}$ signal via a $10 \Omega$ gate resistor.

Fig. 10 shows the circuit waveforms. The MOS-triggered thyristor switches on very fast allowing the voltage on the inductor to rise. The generator is a very weak source and it is fully discharged within $10 \mathrm{~ns}$ of turning the device on. During the charging of the diode, the generator loses $25 \%$ of its charge (and voltage) within 3 ns. Fig. 10 (center) shows the associated current pulse in the device. Fig. 10 (bottom) shows the 

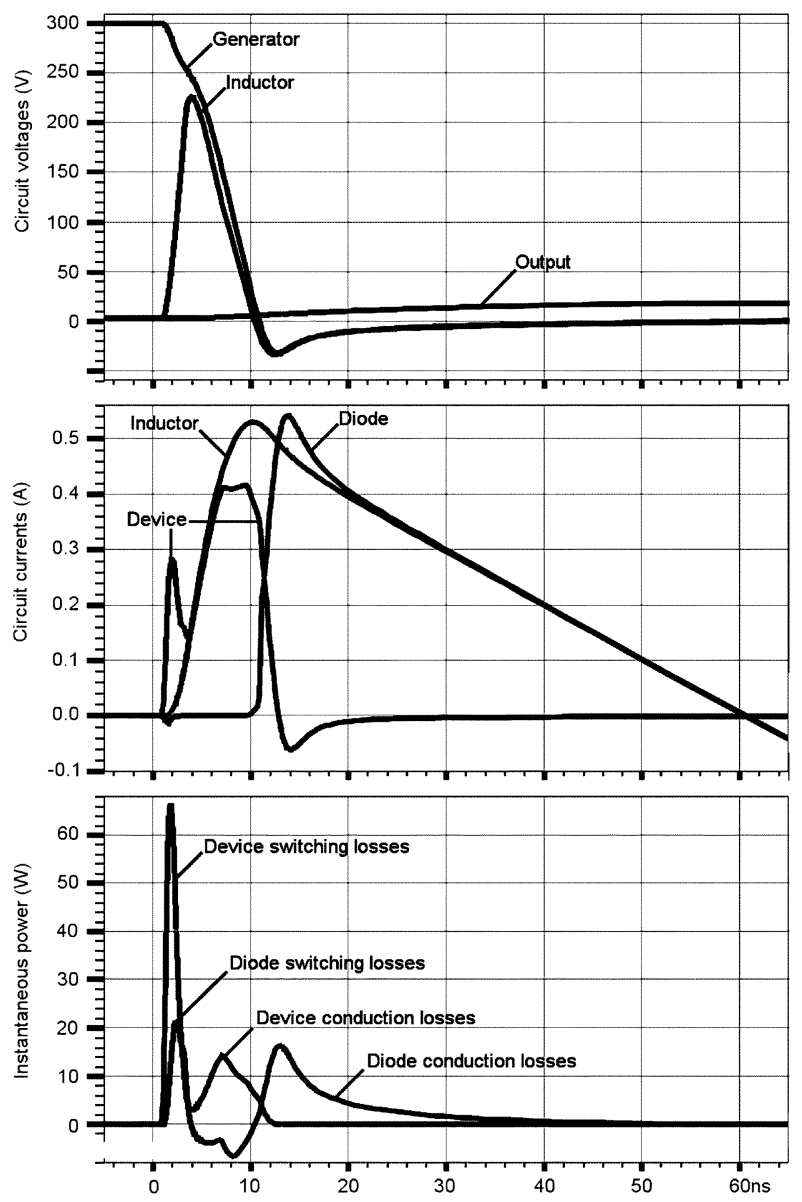

Fig. 10. Circuit switching waveforms using 30-cell MOS-triggered thyristor in single-pulse buck-converter. (Top) Generator, inductor, and output capacitor voltage. (Center) Device (anode), diode, and inductor currents. (Bottom) Device and diode instantaneous powers showing switching and conduction losses.

instantaneous power associated with this current pulse which is relatively high because of the high voltage across the device. Some of the energy lost from the generator is stored in the charged diode.

During the magnetization phase, the inductor current rises and the diode energy is recovered, however the device is conducting an increasing current and incurring conduction losses. The smaller the inductor the higher this current rises, and therefore these conduction losses impose a lower limit for the inductor value.

In the free-wheeling phase, the device current is zero and the diode turns on, showing a combination of turn-on and conduction losses.

\section{B. Device Behavior}

Fig. 11 shows the voltage and current waveforms of the thyristor during conversion. The top graph shows that the device voltage $V_{\mathrm{AK}}$ drops rapidly to around $20 \mathrm{~V}$ then increases and drops again.

The lower graph shows currents through all of the device terminals. After the initial 2 ns delay the thyristor begins to turn on and the anode current peaks at $300 \mathrm{~mA}$ as the diode is charged, the device voltage remaining over $200 \mathrm{~V}$. This is the period of highest losses in the thyristor, see Fig. 10 (bottom). As the diode
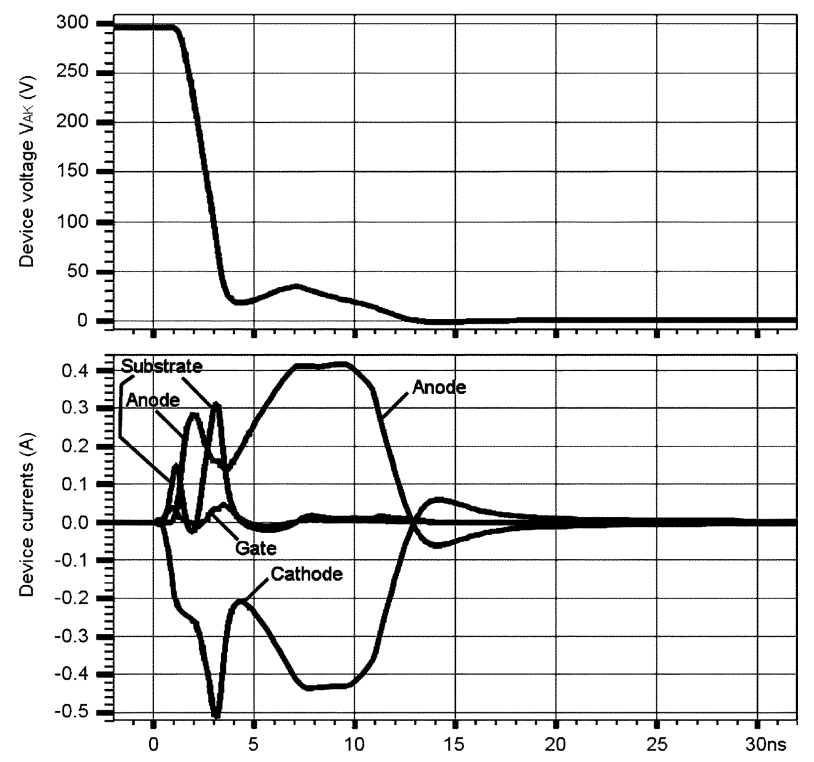

Fig. 11. Expanded device switching waveforms for circuit containing 30-cell MTT. (Top) Device voltage $\left(V_{\mathrm{AK}}\right)$. (Bottom) Device currents.

is charged the anode-cathode voltage drops and the anode current falls. In addition the potential of the devices bulk is rising and this draws a high capacitive substrate current causing further losses. During the magnetization phase the anode current rises to $400 \mathrm{~mA}$. By this time, the carrier concentrations in the thyristor have had time to rise much further and the device voltage is only around $35 \mathrm{~V}$. This equates to $13 \mathrm{~mA}$ per cell, and in steady-state the on-state voltage would be around $2.6 \mathrm{~V}$. During the period where the device current stays constant $(7, \ldots, 10 \mathrm{~ns})$ the device voltage is still dropping, showing that the steady-state carrier distribution has not yet been reached.

In this circuit configuration the gate current is small in comparison to the other terminal currents.

\section{Switching Process of the MOS-Triggered Thyristor}

It is clear, that in the 5-10 ns of conduction required of the switch, the thyristor cannot turn on fully, and the carrier distributions of Fig. 12 show just how far from a steady-state distribution the device actually remains. The carrier distribution in a MOSFET, by comparison, would remain at the background doping level of around $0.06-0.1 \times 10^{17}$, and that of an IGBT would remain tied down at the shorted emitter end. The distributions are taken along a line through the drift region, from the anode to the cathode.

It can be seen that the resistance is highest at the cathode end between 50 and $60 \mu \mathrm{m}$. It is in this region that most of the device voltage is dropped. IGBTs and MOSFETs would have an even lower carrier concentration in this region as there is no injection from the $\mathrm{n}^{+}$-well. We now compare the three devices by running identical simulations with the IGBT and MOSFET.

\section{Comparison MOSFET-IGBT-MOS-Triggered Thyristor}

The device voltage waveforms in Fig. 13(top) appear to show that the thyristor and IGBT switch on faster than the MOSFET. In fact, the MOSFET does initially switch on very fast; however, because the charging current for the diode builds 


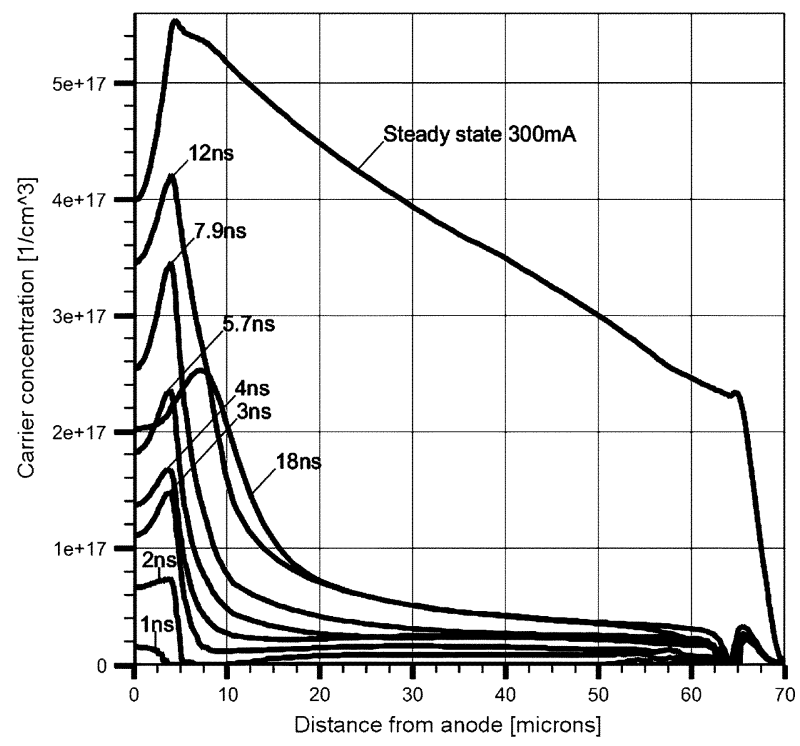

Fig. 12. Simulated carrier concentrations through the 30-cell thyristor during the conversion cycle of Figs. 10 and 11, and the thyristor steady-state distribution at $300 \mathrm{~mA}$.

up very rapidly, the MOSFET almost immediately enters its saturated state. The device is effectively switching into a short circuit. By contrast, the IGBT and thyristor resistances continue to fall as their carrier densities increase, and therefore the device voltages decrease more rapidly in comparison to that of the MOSFET. At approximately 4 to $5 \mathrm{~ns}$ the device voltages swing upward again, reflecting the increasing device currents. The anode current (or collector or drain currents, respectively), shown in Fig. 13 (bottom), contributes to this rising device voltage, but so does the current flowing through the cathode and substrate. This displacement current is large (see Fig. 11) and is caused by the device substrate remaining shorted to ground while the cathode (or emitter or source respectively) potential follows the diode voltage [see Fig. 13 (center)].

\section{Design TRADEOFFS}

\section{A. Generation Efficiency Versus Conversion Efficiency}

In [4] we reported on the influence of device area on generation efficiency. The 30-cell devices absorb around $13 \%$ of the charge from the generator as the voltage is being generated. This imposes an upper limit on the active device area.

Leakage current through the devices was deemed to be insignificant for the device structures proposed here. The converter exhibits leakage mainly during the portion of the flight for which the voltage exceeds $250 \mathrm{~V}$. This time depends mainly on the nature of the motion, and it is usually below $10 \mathrm{~ms}$ for walking-induced motion. For a 30-cell MOSFET this is estimated as a loss of $29.4 \mathrm{pC}$, which is negligible compared to the $3 \mathrm{nC}$ on the generator.

\section{B. Influence of Device Area on Energy Yield}

Although there is no risk of devices overheating, the efficiency is important because the system must generate enough energy for the application from as small a generator footprint
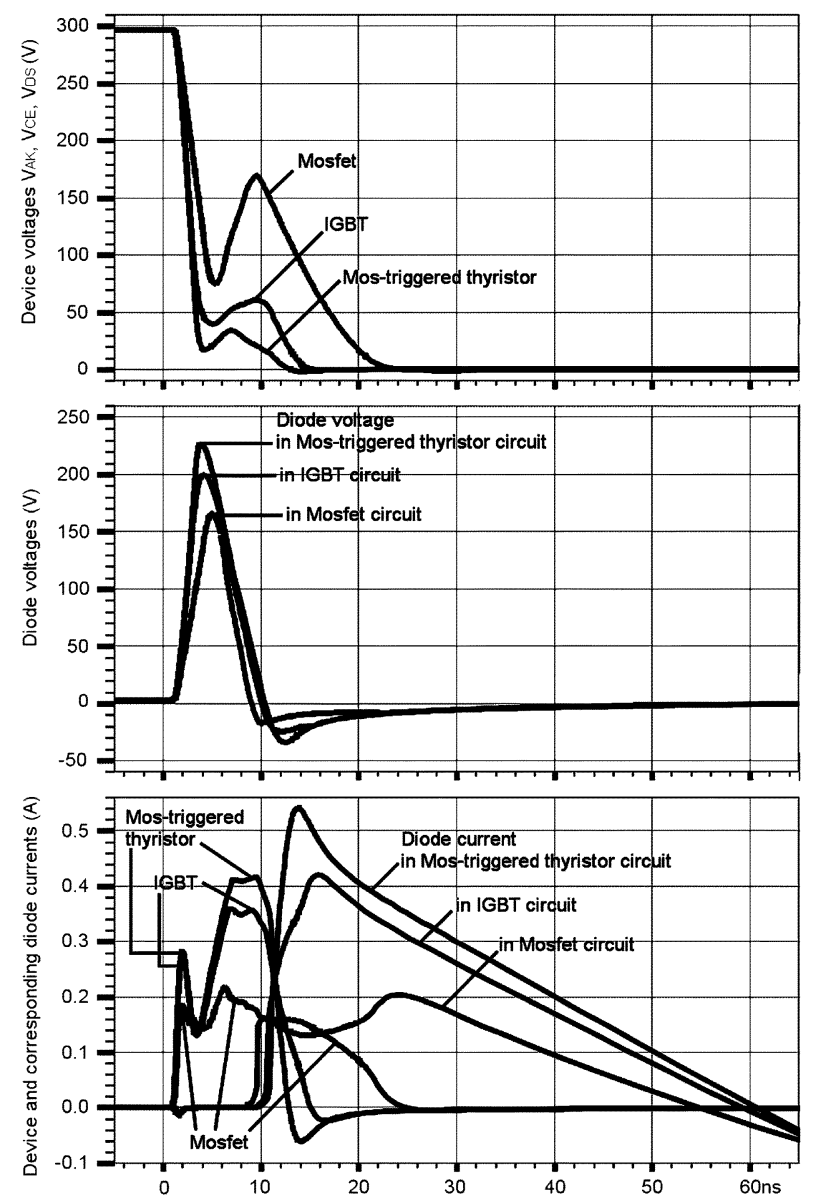

Fig. 13. Comparison of conversion waveforms for thyristor, $I_{G B T}$ and MOSFET circuits. (30-cell devices and diodes). (Top) Device voltages ( $V_{\mathrm{AK}}$, $\left.V_{\mathrm{CE}}, V_{\mathrm{DS}}\right)$. (Center) Diode voltages. (Bottom) Device and diode (anode) currents.

as possible. A typical system would require tens of $\mathrm{nJ}$ to measure, process, and transmit one data point. Generators would probably produce this energy in around 1-10 strokes. Medical applications may have a transfer rate of one eight-bit value per minute, and the higher the system efficiency, the lower the number of strokes required and the smaller the generator can be made.

The high current density device structures have significantly increased the overall generator efficiency for a given size of generator and output inductor. The conversion efficiency was extracted from the mixed circuit device simulations, for a range of device areas and for the three different device structures. The results are shown in Fig. 14. The diode is scaled with the device area.

Considering conversion efficiency only at high device areas there is little to choose between the device structures; the MOStriggered thyristor has not latched up because the current density is too low, so therefore it produces an efficiency which is virtually identical to that of the IGBT. For increasing device sizes, the energy which is available to start with reduces sharply [4].

A clear difference in conduction performance emerges as the device size is reduced. The overall efficiency is still limited by the generation efficiency. At 30 cells the thyristor latches up and is significantly more efficient than the IGBT, which, although 


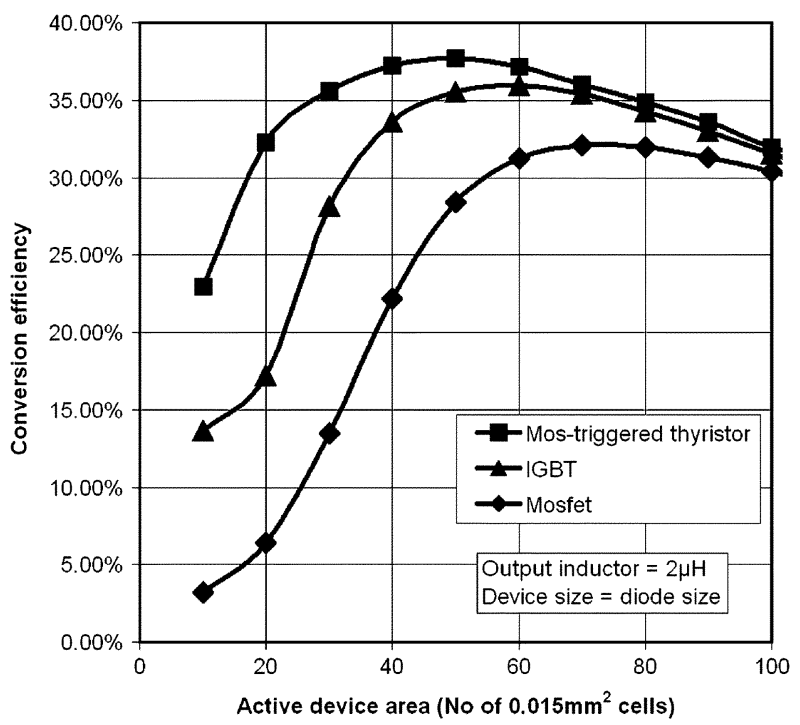

Fig. 14. Summarized simulated conversion results showing efficiency for various device areas during the conversion phase only.

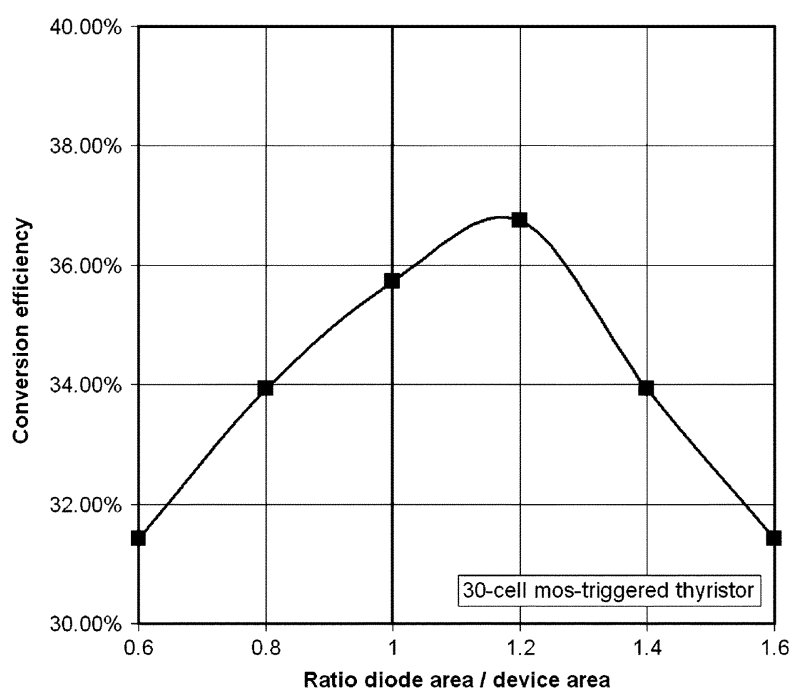

Fig. 15. Simulated conversion efficiency versus ratio of diode/device area.

in saturation, has a far higher efficiency that the fully saturated MOSFET.

The ten-cell IGBT shows an efficiency which is higher than might be expected because the current density is high enough to cause dynamic latch-up.

The curves in Fig. 14 reflect the fact that increasing the device area reduces the conduction losses during the magnetization phase. This requirement, however, is in opposition to the need to reduce the device area in order to increase generation efficiency.

In the free-wheeling phase, there is initially a significant voltage drop $(>20 \mathrm{~V})$ over the diode which reduces as the carrier distribution climbs to its steady-state level. Increasing the diode area would decrease the power loss during this phase but would also increase the turn-on losses in the switching device during the diodes charging phase (see Fig. 4). These device and diode tradeoffs result in the curves of Fig. 14 having a peak efficiency at particular device areas.

\section{Influence of the Diode}

Until now, the diode has been scaled to have the same active area as the switching device. However, when doing so, especially in the MOSFET circuit, the diode losses are far lower than the switch losses. This would appear to suggest that the diode area could be reduced.

In the case of the MOS-gated thyristor, the switch is better matched to the diode and the respective power losses are much closer. This can be seen in Fig. 15, which shows the efficiency of the 30-cell thyristor circuit, as the diode area is varied. At equal areas the circuit is close to its optimum. The diode area is responsible for a significant portion of the device losses. Increasing the diode area from this optimum point increases switch losses due to charge sharing, and reducing the diode area increases the diodes turn-on and conduction losses. Carrying out this scaling on switch and diode pairs in this application is important, and it results in higher efficiency.

It has been seen that the diode is important because the current necessary to increase the reverse bias voltage causes power loss in the switching device and because turn on and conduction losses occur in the free-wheeling period. The diode voltages in Fig. 13 (center) show that the diode never reaches the generator voltage, and its blocking voltage could be designed for $200 \mathrm{~V}$ or even lower. In addition, it takes a certain time for breakdown to occur, and the diode is only ever blocking a high voltage for 1-2 ns at a time. The lower voltage rating of the lateral diode would result in a shorter cell cross section, and the higher conductivity which would allow the cell width to be reduced. A lower voltage diode would therefore be significantly smaller for a given current carrying capacity, which would increase generation efficiency, reduce the initial switch turnon losses and reduce diode turn on and conduction losses.

\section{CONCLUSION}

Switching device customization for self-powered sensors is vital because the switch and diode structures strongly influence the chip area taken up by the generator and inductor. It is important to minimize this area for a given energy yield, as required to transmit a measurement per ten or so generator strokes.

Electrostatic microgenerators require device structures with low parasitic capacitances and ratings of around $300 \mathrm{~V}$. Because of the low value of charge available on the generator, pulse operation of the converter circuit and switching devices can be used, and devices without turn-off capabilities are sufficient. Further, it can be assumed that avalanching is nondestructive. It is therefore possible to optimize the devices turn-on capabilities at the expense of turn off. For the IGBT, the device threshold can be lowered and the latching current reduced to the point where it becomes a MOS-triggered thyristor, and the latching action is used to advantage. Results of finite-element simulation of MOSFET, IGBT, and MOS-triggered thyristor in the full circuit have shown that the thyristor produces the highest overall efficiency and therefore makes best use of the generator. The MOSFET cannot be operated in its linear mode due to the restrictions on its area imposed by restrictions on capacitance, and it remains the least efficient option. 
The diode design is also important because it is responsible for a large fraction of the turn-on loss of the switching device. Its ideal area and optimal blocking voltage are a function of the chosen device structure and respective area.

The study reported here could be extended to a variety of semiconductor processes, including thick SOI, now that it has been established that lateral double-sided-injection turn-on devices are preferable to MOSFETs.

\section{REFERENCES}

[1] P. Needham and L. Gamlyn. Arrhythmia analysis in the community. presented at Proc. Int. Workshop Wearable Implantable Body Sensor Networks. [Online]Available: vip.doc.ic.ac.uk/bsn_2004/program

[2] L. Gamlyn, P. Needham, S. M. Sopher, and T. J. Harris, "The development of a neural network-based ambulatory ECG monitor," Neural Comput. Appl., vol. 41, no. 4, pp. 273-278, Apr. 1999.

[3] S. Roundy, P. K. Wright, and J. M. Rabaey, Energy Scavenging for Wireless Sensor Networks. Norwell, MA: Kluwer, 2003.

[4] B. H. Stark, P. D. Mitcheson, P. Miao, T. C. Green, E. M. Yeatman, and A. S. Holmes, "Power processing issues for micropower electrostatic generators," in Proc. 35th IEEE Power Electronics Specialists Conf., Jun. 2004, pp. 4156-4162.

[5] T. von Beuren, P. D. Mitcheson, T. C. Green, E. M. Yeatman, A. S. Holmes, and G. Troester, Optimization of inertial micropower generators for human walking motion, in IEEE Sensors J.. accepted.

[6] P. D. Mitcheson, T. C. Green, E. M. Yeatman, and A. S. Holmes, "Architectures for vibration-driven micropower generators," IEEE/ASME J. $M E M S$, vol. 13, no. 3, pp. 429-440, Jun. 2004.

[7] P. D. Mitcheson, B. H. Stark, P. Miao, E. M. Yeatman, A. S. Holmes, and T. C. Green, "Analysis and optimization of MEMS electrostatic on-chip power supply for self-powering of slow-moving sensors," in Proc. 17th Eur. Conf. Sensors Actuators, Guimarães, Portugal, Sep. 2003, pp. 492-495.

[8] S. Meninger, J. O. Mur-Miranja, R. Amirtharajah, A. P. Chandrakasan, and J. H. Lang, "Vibration-to-electric energy conversion," IEEE Trans. Very Large Scale (VLSI) Syst., vol. 9, no. 1, pp. 64-67, Jan. 2001.
[9] B. H. Stark, P. D. Mitcheson, P. Miao, T. C. Green, E. M. Yeatman, and A. S. Holmes, "Converter circuit design, semiconductor device selection and analysis of parasitics for micropower electrostatic generators," IEEE Trans. Power Electron., submitted for publication.

[10] T. Letavic, J. Petruzzello, M. Simpson, J. Curcio, S. Mukherjee, J. Davidson, S. Peake, C. Rogers, P. Rutter, M. Warwick, and R. Grover, "Lateral Smart-Discrete Process and Devices based on Thin-Layer Silicon-on-Insulator," in Proc. IEEE ISPSD, Osaka, Japan, 2001, pp. 407-410.

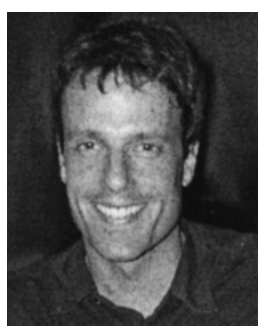

B. H. Stark received the Diploma degree in electrical engineering from the Swiss Federal Institute of Technology, and the Ph.D. degree in engineering from Cambridge University, Cambridge, U.K. in 1995 and 2000, respectively.

He was a Junior Research Fellow at St. Hughs College, Oxford, U.K., until 2000 and is currently a Member of the Control and Power Group, Imperial College, London, U.K. His academic interests include the application of power electronics to energy generation.

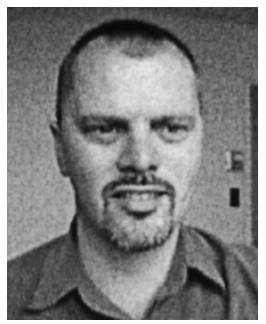

T. C. Green received the B.Sc. (Eng.) from Imperial College, London, U.K. and the Ph.D. degree from Heriot-Watt University, Edinburgh, U.K., both in electrical engineering, in 1986 and 1990, respectively.

He was a Lecturer at Heriot-Watt University until 1994 , and is now a Reader at Imperial College, London, and Deputy Head, Control and Power Research Group. He has research interests in power electronics applied to generation and distribution of energy, including issues of renewable and distributed generation, microgrids, power quality, active power filters, and flexible ac transmission systems. 